\title{
A simple electronic device for time-lapse recording of neural and other cell movements using a home video cassette recorder
}

\author{
Dirk P. Struwe ${ }^{1}$, Zheng Wang ${ }^{3}$, Adriaan Droog ${ }^{1}$, Marja van Graft ${ }^{2}$, Marga Deenen ${ }^{3}$, \\ Enrico Marani ${ }^{3}$ and Dirk L. Ypey ${ }^{1,3}$ \\ Departments of ${ }^{1}$ Electrical Engineering, Biomedical Division and ${ }^{2}$ Applied Physics, Cell Characterization Group, University of \\ Twente, 7500 AE Enschede (The Netherlands) and ${ }^{3}$ Department of Physiology, Leiden University, 2300 RC Leiden (The Netherlands)
}

(Received 12 February 1992)

(Revised version received 23 June 1992)

(Accepted 26 June 1992)

Key words: Time-lapse recording; Video micrography; Home video cassette recorder; Cell movement; Growth cone; Myelination; Dorsal root ganglion; Natural killer cells

This article describes a simple electronic unit to obtain time-lapse recordings with the use of a common remote-controlled home video cassette recorder, for example a VHS recorder. The electronic unit is a timer to be connected to the remote-control unit. The video cassette recorder itself remains unchanged. Replay of the recorded images speeds up the original process by a factor of $2-100 \times$ or more. This technique has been applied in video micrographic studies of (1) the development of dorsal root ganglion (DRG) cells in culture, including growth cone and Schwann cell movements, and (2) tumor cell killing by natural killer (NK) cells.

\section{Introduction}

In studies of movement, development and differentiation of cells in culture it is very useful to make micrographic recordings which can be replayed at increased speed. Time-lapse cinemicrography is often used by applying a film camera for this purpose. While this results in high-quality photographic pictures, they cannot be seen immediately after exposure. Special video cassette recorders (VCRs) are commercially available for time-lapse exposure. They are very expensive and,

Correspondence: Dirk L. Ypey, Ph.D., Department of Electrical Engineering, Biomedical Engineering Division, University of Twente, P.O. Box 217, 7500 AE Enschede, The Netherlands. Tel.: (31-53) 89-2760; FAX: (31-53) 32-8439. furthermore, are not designed to switch the microscope illumination.

To overcome these problems, we have used a common home VCR connected to a simple electronic unit serving as timer controlling the recorder. Recording occurs during short periods (approx. $1 \mathrm{~s}$ ) separated by an adjustable pause. The timer determines the duration of the recording period and subsequent pause. The ratio of recording to recording plus pause defines the factor by which the original recording time is reduced on playback. An important additional advantage of this technique is that the timer can also be used to keep the light source of the microscope switched on during the recording period only. This is essential when culturing cells in a medium that is degraded under the influence of visible light.

During a computer search we did not find our electronic unit published in the scientific litera- 
ture. 'The present device has already been published in abstract form (Struwe et al., 1991).

\section{Description}

In principle, time-lapse recordings can be made by hand with a remote-controlled normal VCR. In this case, one has to press repetitively the PLAY and PAUSE switches of the remote-control unit while the VCR is in the RECORD mode. Upon replaying the record, the sequence of recorded episodes is displayed free of irregular switch-on and switch-off movements. Only the small jerky movements of the object indicate that a time-lapse recording is being replayed.

We automatized the method described above by connecting the remote-control unit of an ordinary VHS VCR to a simple electronic timer (Fig. 1). Electrically operated switches (relays) inside the timer are simply connected in parallel with the PLAY and PAUSE switches of the remotecontrol unit of the VCR. The timer circuitry controls these switches. The electronic circuit diagram of the timer used is presented and described in the Appendix.

Nothing has to be changed in the electronics of the remote-control unit; only wires have to be connected to the switches, which can easily be done. The VCR itself remains unchanged. The timer may be set, for example, for record periods of about $1 \mathrm{~s}$ and for pause periods of about $90 \mathrm{~s}$. Microscope illumination is also controlled by the timer (Fig. 1). The cell culture in its light-sensitive medium is then illuminated only during the short record periods.

Video recording of fluorescently labeled cells may require an $\mathrm{Hg}$ lamp. As this lamp cannot be switched, an electrically operated shutter in the excitation light path must be applied. We used the same adapted remote-control unit to control

\footnotetext{
${ }^{1}$ Recently we found a unit for time-lapse recording with a camcorder in a Dutch amateur electronic magazine (Elektuur, Ph. Bosma, March 1990, pp. 44-46). However, it was based on a different circuit and did not have the option to switch the microscope lamp.
}

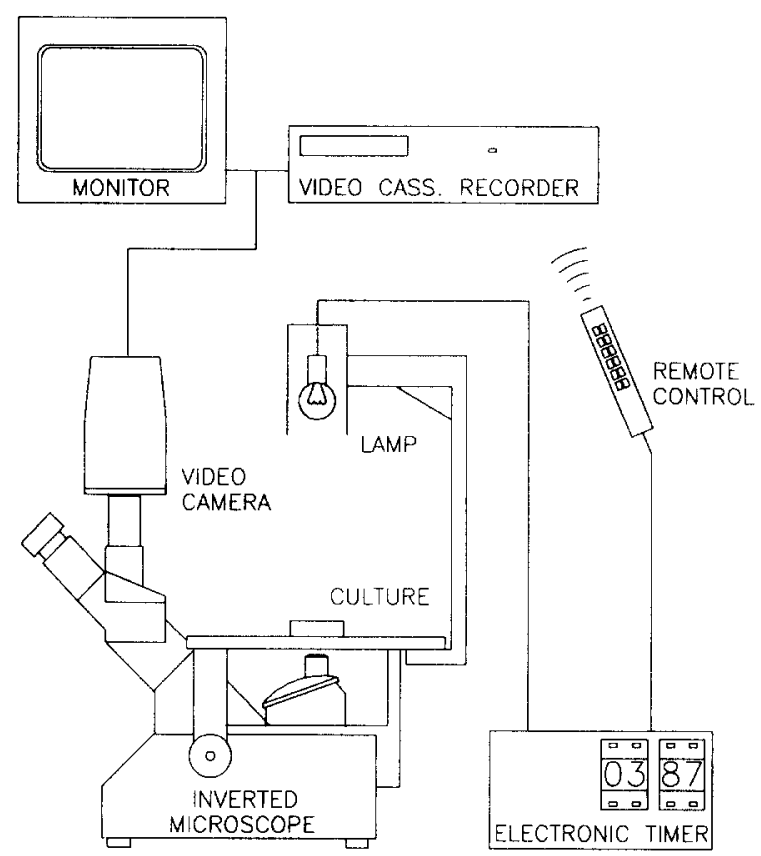

Fig. 1. Set-up for time-lapse video micrography.

3 different types of JVC VCRs (types HR-D170E, HR-S5000E and HR-D250E).

To make a time-lapse recording, the timer has to be set for a certain (short) PLAY and (longer) PAUSE duration. Then, the VCR has to be set in the record mode. Subsequently, the timer has to be started. The time-lapse box switches the microscope lamp on at the beginning of the PLAY period, but the VCR is set in the RECORD mode a few seconds (adjustable) later to allow the lamp time to reach its full brightness. In addition, the VCR needs about $1 \mathrm{~s}$ to start a recording. As a result, for example, a selected PLAY duration of $3 \mathrm{~s}$ will result in a recording period of about $1 \mathrm{~s}$.

Selection of a 3-s PLAY duration and an 87-s PAUSE duration will result in records of approximately $1 \mathrm{~s}$ every $90 \mathrm{~s}$. The effective acceleration on replay can be calibrated by a record of a clock running a few minutes.

Instead of controlling the PLAY and PAUSE switches in the record mode of the remote control, the RECORD and STOP switches can be used for time-lapse recording. The small disadvantage is that the VCR needs more time for 
starting a recording because the tape is taken from the video head drum upon closing the STOP switch. However, the advantage is that there will be less wear of the tape and video head drum. With our VCRs (JVC-types HR-D170E and HRS5000E) good results were obtained without flickering.

For video micrography, the video camera has to be coupled to the microscope (Fig. 1). Ideally, a microscope with a trinocular tube or side camera port (in the case of an inverted microscope) is used together with a photo eye-piece. Thus the video camera is used without camera lens and is coupled to either the photo tube or side port of the microscope. Usually a video camera with a thread-mount lens connector, so-called C-mount, is used. Most microscope manufacturers provide a C-mount adapter to couple a $\mathrm{C}$-mount camera to the microscope. However, one can even position the video camera close to one of the eyepieces of the binocular eye-piece tube. The following combinations will work: (1) a camera with lens (opened aperture diaphragm) and a (normal or photo) eye-piece in the tube; (2) a camera without lens and a (normal or photo) eye-piece in the tube; or (3) a camera without lens and the eye-piece removed from the tube.

The time-lapse set-up is represented in Fig. 1. An inverted microscope (Nikon Diaphot) was used, in our situation part of a patch-clamp set-up. We used a $10 \times$ phase-contrast objective (Nikon CF DL-10 $\times$ ) in combination with a $5 \times$ photo eye-piece (Olympus photo eye-piece NFK $5 \times$ LD) in the side camera port. The video camera (Bosch T6XK921 A1) without lens was attached to the side camera port of the microscope with a Cmount adapter. The combination of a $10 \times$ objective, $5 \times$ photo eye-piece and a 1 -in camera tube results in an object field of approximately $240 \times$ $160 \mu \mathrm{m}$ on the monitor $(1750 \mu \mathrm{m}$ in the observation turret with CFW $10 \times$ eye-pieces). ${ }^{2}$

\footnotetext{
2 Today, many cameras use a $2 / 3$-in CCD pick-up device with a sensitive area of $8.8 \times 6.6 \mathrm{~mm}$. For instance, a Sony DXC $115 \mathrm{P}$ camera, in combination with a $10 \times$ objective and an Olympus NFK $5 \times$ LD photo eye-piece in the Nikon Diaphot, gives an area of $140 \times 110 \mu \mathrm{m}$ on the video monitor.
}

In general, a greater object field may be preferred, using a photo eye-piece with lower magnification or a $4 \times$ phase-contrast objective. A 1 -in camera tube has about a 16 -mm-diagonal sensitive area $(9.6 \times 12.8 \mathrm{~mm})$ which is somewhat less than the diameter of the primary image plane (where a graticule can be laid) of a wide-field eye-piece $(18$ or $20 \mathrm{~mm})$. Therefore, a $1 \times$ photo eye-piece will result in a somewhat smaller object field for the camera (with 1-in camera tube) than seen in the observation turret.

The video camera output is connected to an ordinary VHS recorder while the picture can be followed on a video monitor. Our electronic timer is connected to the remote-control unit of the VCR as well as to the microscope illumination to keep the lamp switched on during the recording period only.

\section{Applications}

Cell movements in dorsal root ganglion cell cultures

We have applied the time-lapse recording technique described mainly to the study of the interactive development of cultured neonatal dorsal root ganglion (DRG) cells in a chemically defined culture medium. DRGs were removed from 1-4 day-old Wistar rats, mechanically dissociated into the constituent cells and cultured in the chemically defined medium R12 (Romijn et al., 1984), supplemented with $10 \mathrm{ng} / \mathrm{ml}$ nerve growth factor $(2.5 \mathrm{~S}-\mathrm{NGF}$, from mouse submaxillary glands, Sigma Chemical Co., St. Louis, MO, USA).

The ganglia dissection and cell culture method is as previously described (Van Dorp et al., 1990) but, for mechanical dissociation of the DRGs, we used a simple micro-dissociation technique similar to that of Sensenbrenner et al. (1969). One to 5 DRGs were directly placed in 3 drops of R12 medium on a poly-D-lysine hydrobromide (mol. wt.: 70,000-150,000; Sigma, $0.1 \mathrm{mg} / \mathrm{ml}$ ) coated coverslip in a $35-\mathrm{mm}$ petri dish. Then, fine pairs of tweezers were used to disrupt the capsules of the ganglia and to dissociate the cells (neurons, glial cells and fibroblasts) by trituration. After a 4-h incubation in the $5 \% \mathrm{CO}_{2}$ incubator $\left(37^{\circ} \mathrm{C}\right.$, 
$100 \% \mathrm{H}_{2} \mathrm{O}$ ) the cells were firmly enough attached to the glass to complete the medium volume to $1 \mathrm{ml}$ in order to continue culturing for 1-12 days (Van Dorp et al., 1990). The medium was refreshed every 2-3 days.

For time-lapse video recording and observation, a culture dish was transferred to a micro$37^{\circ} \mathrm{C} / \mathrm{CO}_{2}$-incubator (Ince et al., 1983) on the stage of an inverted phase-contrast microscope. Time-lapse recordings were made during 1-96 h. The time-lapse control box was set for 4-s PLAY and 99-s PAUSE durations.

Here, we illustrate growth cone advancement during nerve process formation and elongation from the cell body of a DRG neuron in the first hours after isolation (Fig. 2). In addition, we show dynamic glial cell changes and interactions during myelination (Fig. 3) in the later stages of DRG cell culture development.

The first photograph of the growth cone timelapse series (Fig. 2) shows the reformation of a growth cone from a varicosity, which is probably a retracted cone formed after the initial outgrowth from a neuronal cell body. In a previous study (Van Dorp et al., 1990) it was demonstrated using immunocytochemistry for neurofilament that this type of cell is a neuron. The intervals between the photographs are approximately 8 min which implies (see calibration bar) that the growth cone advances with an approximate speed of 1 $\mu \mathrm{m} / \mathrm{min}$. Although the mean direction of growth is quite linear in this case, the growth cone is changing its shape and direction continuously.

The myelination time-lapse series (Fig. 3a-d) shows a presumed glial cell retracting from an myelinating network (a, left side), contracting (b), dividing (c) and separating (d), while the network is remodeled continuously. Actual cell division occurs rapidly (within $10 \mathrm{~min}$ ). The cell considered as well as the others of this sequence are not

Fig. 2. Photographs taken from a video monitor of a time-lapse recording: formation and growth of a growth cone from a varicosity after the initial outgrowth from a neuronal cell body. This cell type is neurofilament positive. Intervals between the photographs are approximately $8 \mathrm{~min}$. The growth cone advances with an approximate speed of $1 \mu \mathrm{m} / \mathrm{min}$. Calibration bar: $50 \mu \mathrm{m}$.
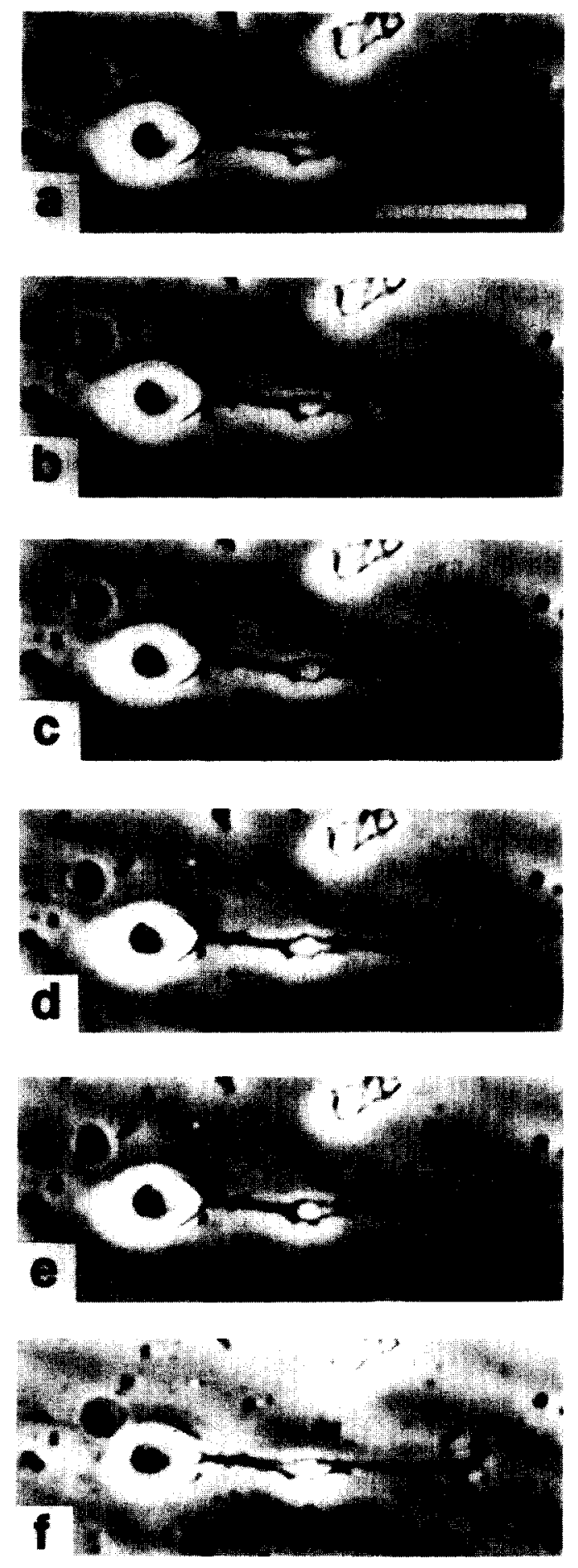
neurofilament-positive type cells (neurons) (Van Dorp et al., 1990). Bright bundles like that labeled ' $m b$ ' have been shown by us (data not shown) to contain neurofilament-positive nerve processes covered by Schwann cell bodies and processes identified with the monoclonal antibody Rat 401 (Friedman et al., 1990). The cell processes of the network (probably myelinating) on the left side of the photographs are in continuous interaction with the bundle ' $m b$ '. The iso- lated Schwann cells clustered on the right side of the photographs are continuously moving until they become aligned with nerve process bundles. The black fiber ('ub') is probably an unmyelinated bundle in which myelination is just starting (see aligned Schwann cell bodies in the top of the photographs).

From this type of time-lapse recording we learned that myelination is a dynamic process of formation and breaking cell-cell contacts.
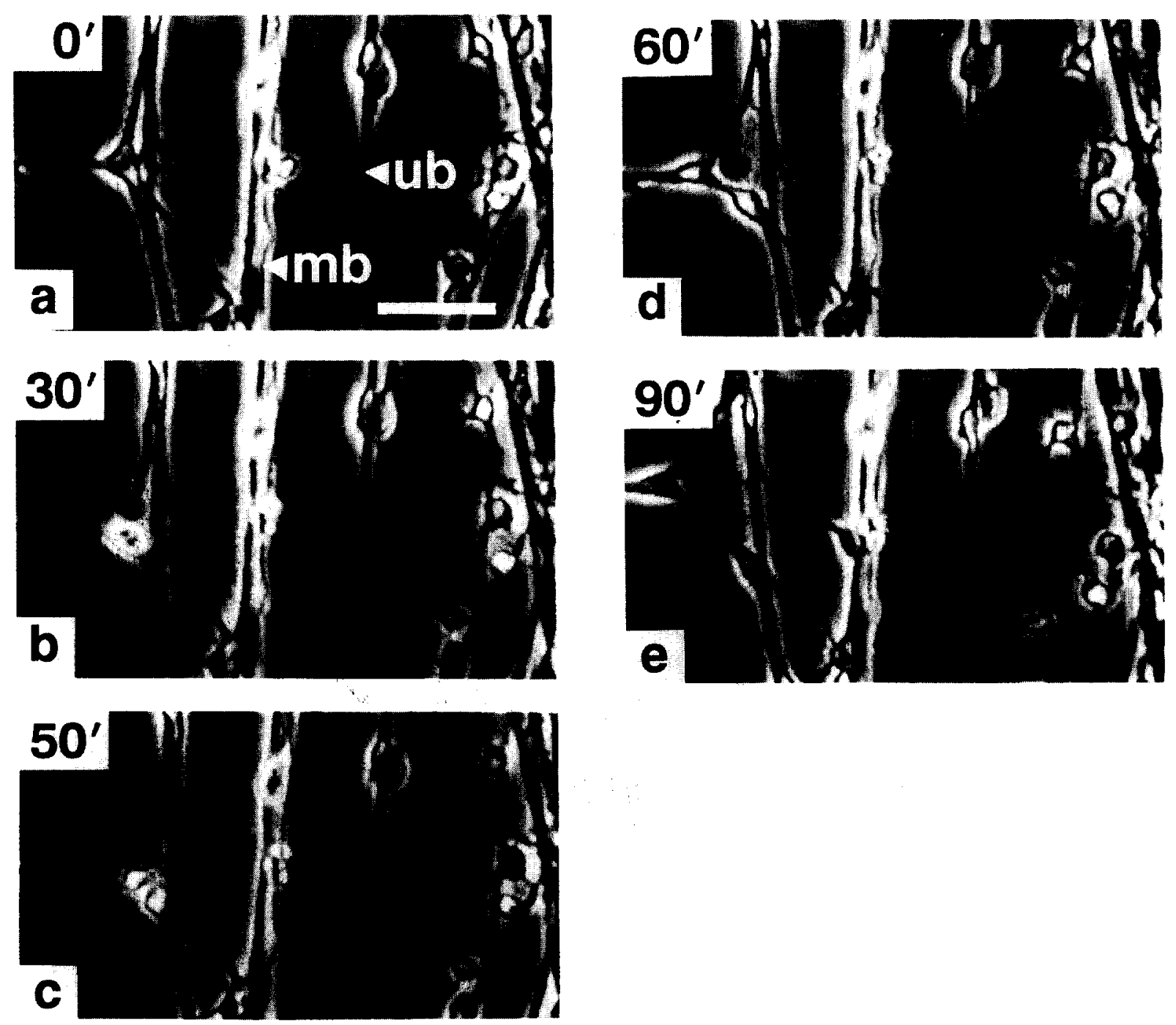

Fig. 3. Photographs taken from a video monitor of a time-lapse recording. Left: cell division of a presumed glial cell. This type of cell is not neurofilament positive. The cell processes of the network on the left side are in continuous interaction with the bright Schwann-cell covered bundle ' $m b$ ' in the middle. Clustered Schwann cells at the right are moving to the left. In the dark fiber 'ub', myelination is probably just starting (Schwann cell bodies in the top). Calibration bar: $50 \mu \mathrm{m}$. 
Cytotoxic interaction between a natural killer (NK) cell and target cell (TC)

The present time-lapse recording technique has also been used to observe the interaction between a natural killer (NK) cell and an appropriate tumor target cell (TC) during patch-clamp studies (Fig. 4).

NK cells were kept in RPMI 1640 without phenol red (Seramed, Berlin, Germany) and cultured as described before (Bolhuis et al., 1984). Cells from the human leukemia cell line K562 were used as TC. These cells were cultured in RPMI 1640 supplemented with penicillin G (100 $\mathrm{U} / \mathrm{ml})$ and streptomycin $(100 \mu \mathrm{g} / \mathrm{ml}$, both from Sigma), L-glutamine (2 Mm) and bicarbonate (2.4 mM) (both from Merck, Darmstadt, Germany) and $10 \%$ heat-inactivated fetal calf serum (Gibco Europe b.v., Breda, The Netherlands). This medium was used for the experiments as well

An experiment was started by placing $2 \times 10^{5}$ $\mathrm{K} 562 \mathrm{TCs}$ in a culture dish which was placed in a micro-incubator on a microscope stage, using a poly-L-lysine hydrobromide (mol. wt.: approx. 150,$000 ; 0.001 \%$ in phosphate-buffered saline, from Sigma) coated microscope coverglass as a bottom of the dish (effective diameter: $19 \mathrm{~mm}$ ) (Ince et al., 1983, 1985). After 15-min incubation at room temperature the K562 cells were attached to the bottom. Thereafter, the same number of NK cells was pipetted into the culture dish.
With this amount of cells, a monolayer of non-adjacent cells was obtained. The cells were observed with phase-contrast microscopy and appeared as spheres with a bright halo.

Several minutes after the heat control was switched on, NK cells started to form a pseudopod and move around, while the K562 TCs remained round and immobile. When an NK cell reached a TC two things could happen. First, the NK cell might interact with the TC without further affecting it (Fig. 4a). However, if an NK cell met an appropriate TC, several morphological changes were observed in both the NK cell and TC. At first a tight bond was formed between the NK cell and TC. During this phase the NK cell was rounding up and attaching firmly onto the TC. The cells could then be observed as 2 distinct round cells with a bright halo, typical for phasecontrast microscopy, at the site of adhesion. Several minutes later this bright halo started fading away, which meant that a tight connection was being established between the NK cell and TC (Fig. 4b). A few minutes later membrane blebs appeared and disappeared at different sites of the TC (Fig. 4c). This lasted for $10-30 \mathrm{~min}$. During this phase the NK cell could hardly be distinguished from the TC. After about $30 \mathrm{~min}$ bleb formation stopped. The TC had a swollen appearance, with a clear cytoplasm and a condensed nucleus surrounded by granular material
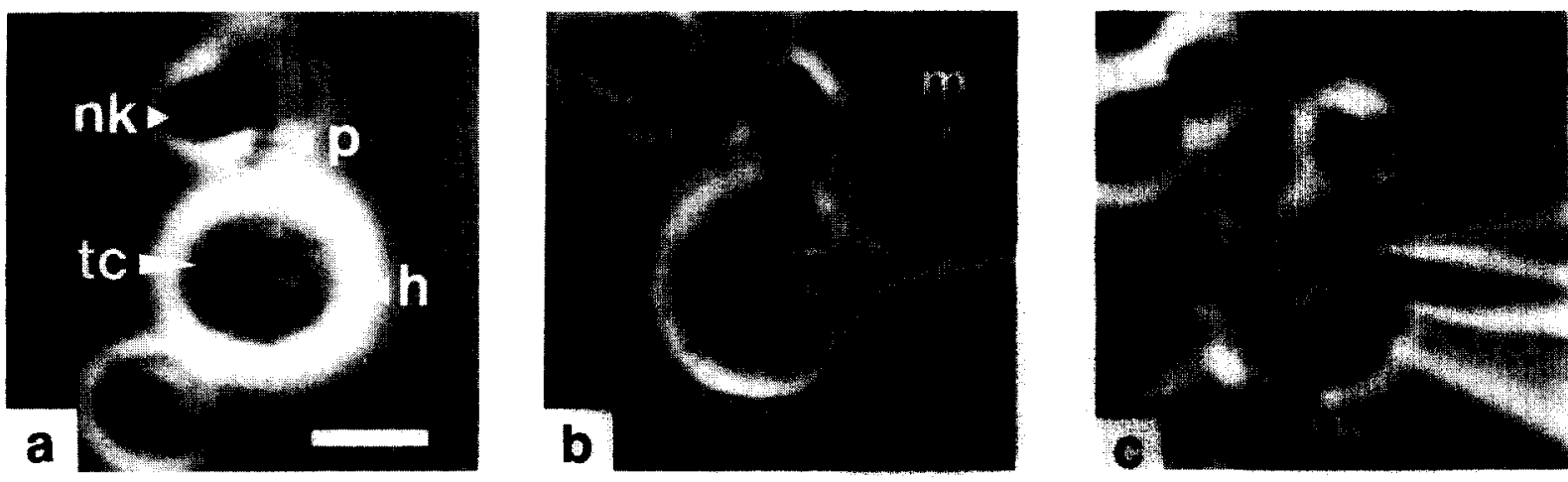

Fig. 4. Photographs taken from a video monitor of a time-lapse recording of the interaction between a natural killer (nk) cell and a K562 target cell (tc) during a patch-clamp study. a: the NK cell has formed a pseudopod (p) and is interacting with a TC. Notice the bright halo $(h)$ on the interaction site of the cells. b: the NK cell is firmly attached onto the TC. The bright halo has disappeared. A glass micropipette $(\mathrm{m})$ has been placed on the surface of the TC for patch-clamp measurements during cytotoxic interaction. $\mathrm{c}$ : at different sites of the TC blebs (b) are occurring. Calibration bar: $10 \mu \mathrm{m}$. 
making brownian-like movements. Then, the NK cell rounded up and the bright halo between the cells could again be observed. The NK cell might detach from the TC and attack another TC.

In our study patch-clamp experiments were performed on either an NK cell or a K562 cell during the cytotoxic process. In these studies it is important to relate membrane electrophysiological changes to morphologic changes in the cells involved in the cytotoxic interaction. The overall cytotoxic process lasts for about $60 \mathrm{~min}$, depending on temperature, $\left[\mathrm{Ca}^{2+}\right]_{o}$, active state of the NK cells and sensitivity of the TCs (Trinchieri, 1989). Therefore, the present time-lapse recording device is very useful because it allowed us to obtain compressed time-controlled, video registrations of the cytotoxic process during the patch-clamp studies.

\section{Discussion}

The applied time-lapse method has been tested only with VHS VCRs. With these recorders, the video head drum remains spinning during the pause period when the PAUSE-PLAY method is used. It thus spins continuously throughout the entire time-lapse period. This should in principle result in increased abrasion of tape and video head drum. We therefore recommend the use of high-quality tape. However, we applied the method for several hundred hours of time-lapse recording without encountering problems. There has so far been no need for servicing the VCRs. Alternatively, when the REC-STOP method is used, the tape is taken away from the video head drum and the drum stops spinning during the pause period. In this case there will be less abrasion of tape and video head drum.

In our set-up, the microscope lamp can be controlled by the time-lapse control box. Frequently switching the lamp on and off decreases its life expectancy. With the halogen lamps we used this was no problem. However, it seems safe to replace an old bulb with a new one when starting a time-lapse recording of long duration. We switched the power of the lamp because this is the most simple solution, but it is also possible to control an electrically operated shutter with the time-lapse box.

Today, camcorders are available with an integrated option which allows time-lapse recordings to be made the same way we did without using a time-lapse control box. However, in this case, one cannot control the microscope lamp and would then also prefer a camcorder with a $\mathrm{C}$-mount lens. Only a few of these camcorders are commercially available.

\section{Acknowledgements}

We thank Dr. S. Hockfield (Section of Neuroanatomy, Yale University School of Medicine, New Haven, CT) for kindly providing us with the Schwann cell antibody Rat 401. The NK cell clone was kindly provided by Dr. R.L.H. Bolhuis from the Radiobiological Institute TNO, Rijswijk, The Netherlands. M.v.G. was supported by a grant from The Netherlands Organization for Scientific Research (NWO) through the Foundation for Biophysics. We thank Professor Dr. J. Greve for support.

\section{Appendix}

\section{The timer}

The schematic diagram of the timer is presented in Fig. 5. The 8-bit comparator U4 compares the counter value of an 8-bit counter, composed of 4-bit counters U5 and U6, to a preset value, selected by U8b, U2 and U3. Thumb wheel switches Sw1-8 and Sw9-16 select PLAY and PAUSE duration, respectively. The counter is incremented by clock pulses from U9. The system clock is adjusted to $1 \mathrm{~Hz}$ with $\mathrm{P} 1$. A (low-active) pulse NTIME is generated when the counter value reaches the preset value (U4 and U7). This results in: resetting of U5 and U6, reversing the level of SWEN and NSWEN (which results in selecting the other preset value for the comparator), exciting of the PLAY or PAUSE relay during a short time (by U8B and U10A), and selecting a current trough LEDs D2 or D3 (by U8B and U10B). Excitation of the PLAY relay is de- 


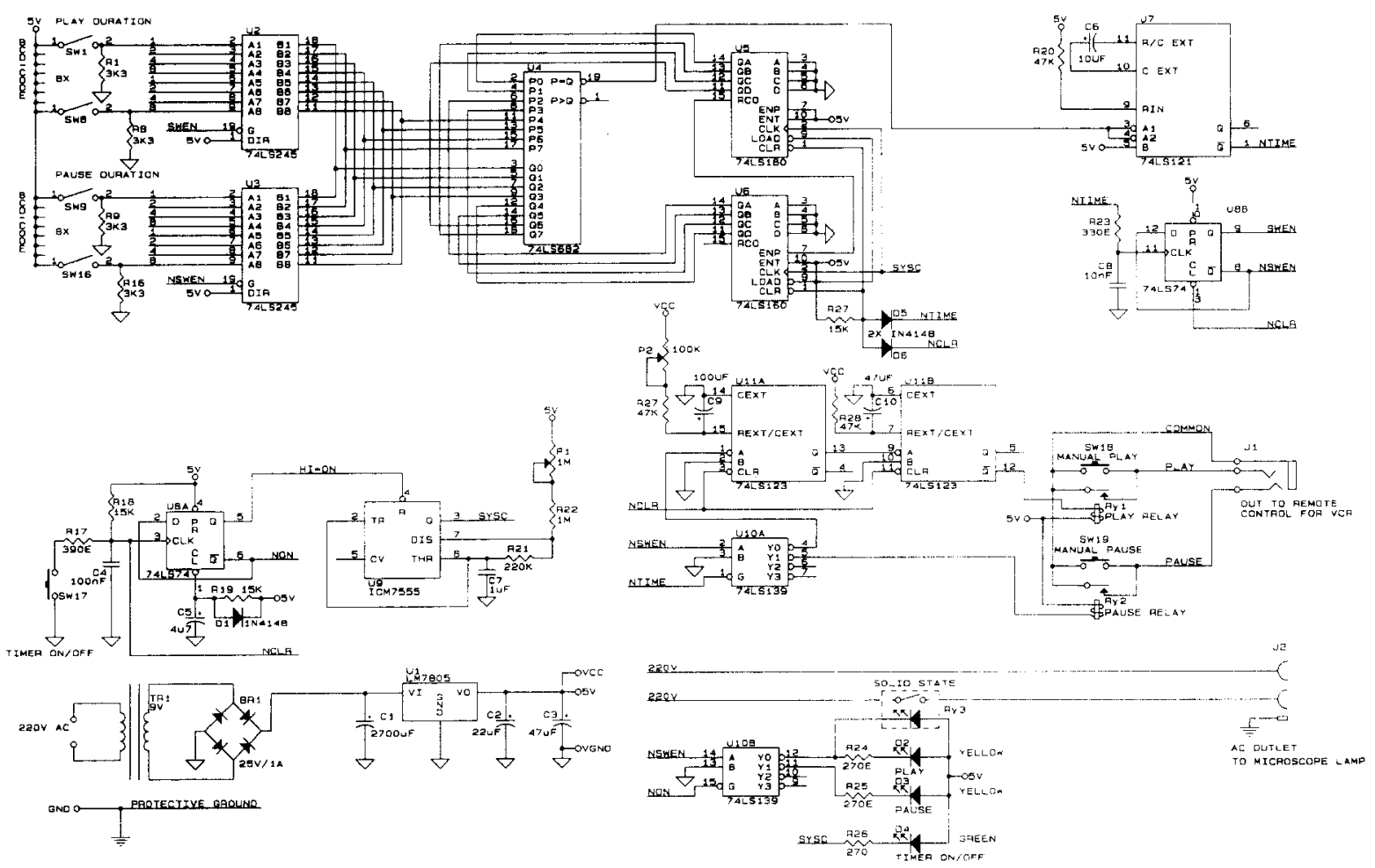

Fig. 5. Schematic diagram of the timer.

layed about $2 \mathrm{~s}$ by U11A and B (adjustable with P2) to allow the lamp to reach its full brightness $(\Delta t$ in Fig. 6).

Solid-state relay Ry3 is connected in parallel to PLAY-LED D2 and controls microscope illumination. Sw17, with U8A, serves as a toggle switch to choose between automatic or manual operation of the remote-control unit (timer on, timer off, respectively). The remote control can always be controlled manually by its original

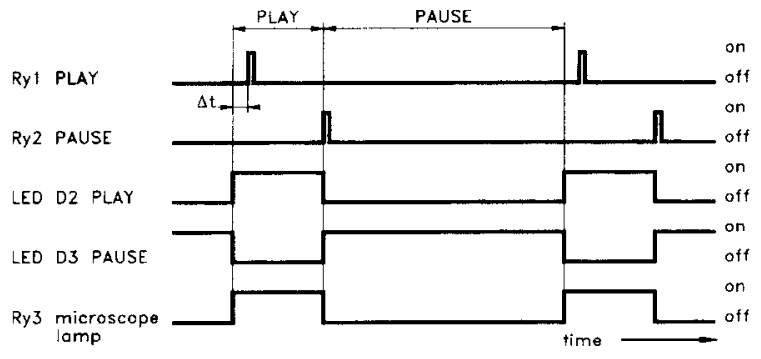

Fig. 6. Timing diagram of relays Ry1, Ry2, Ry3 and LEDs D2 and D3. switches or by Sw18 and Sw19. LED D4 flashes with the clock frequency when the timer is on, but lights up continuously when the timer is off. LEDs D2 and D3 do not light up when the timer is off (by signal NON).

Upon switching the power on, U8A is reset and the timer switches off. After switching the timer on with SW17, the first period is a PLAY period. The timing diagram is presented in Fig. 6.

Abbreviations used in the circuit diagram are: SWEN = switch enable; NTIME $=$ not-time; NSWEN $=$ not-switch enable; NCLR $=$ not-clear; SYSC $=$ system clock: NON $=$ not-on.

The VCR needs a certain time to start a recording after a PAUSE period (approx. $1 \mathrm{~s}$ for JVC types HR-D170E and HR-D250E, a fraction of a second for newer types like HR-S5000E). Thus, when a 2-s lamp pre-glow period is chosen (selected with $\mathrm{P} 2$ ), the effective recording duration is approx. $3 \mathrm{~s}$ shorter than the preset PLAY period. The maximal PAUSE period appears to be about $320 \mathrm{~s}$, since after a longer pause the VCR automatically stops. Alternatively, if the 
RECORD-STOP option is used, the pause duration is no longer limited.

However, with our timer, the maximal PAUSE duration is limited to $99 \mathrm{~s}$ (when the system clock is at $1 \mathrm{~Hz}$ ). On playback, the original process is speeded up about $100 \times$. When a longer PAUSE period is preferred, one can change the clock frequency to $0.5 \mathrm{~Hz}$ (acceleration approx. $200 \times$ ). Otherwise the circuit with comparator, counters and preselection can be extended to 12 bit (U4, U5, U6, U2, U3, Sw1-Sw16).

\section{Adaptation of the remote-control unit}

Reed relays Ry1, Ry 2 as well as switches Sw18, Sw19 have to be connected in parallel to the original PLAY and PAUSE switches in the remote-control unit of the VCR. For our JVC recorders an integrated circuit (Mitsubishi type M50115 AP) is used in the remote-control unit; pins 13 and 15 are connected for PLAY and pins 13 and 16 are connected for PAUSE. In this case a 3-pole miniature stereo headphone plug can be used for interconnection. When a programmable remote control is used, i.e., a remote control which can 'learn' the code of another remote control, one adapted version can be used together with many different types of VCRs. Furthermore, one can then easily change from the PLAY-PAUSE to the RECORD-STOP option.

\section{References}

Bolhuis, R.L.H., van de Griend, R.J. and Ronteltap, C.P.M. (1984) Clonal expansion of human B73.1-positive natural killer cells or large granular lymphocytes exerting strong antibody-dependent and -independent cytotoxicity and occasionally lectin-dependent cytotoxicity. Nat. Immun. Cell Growth Regul., 3: 61-72.

Friedman, B., Zaremba, S. and Hockfield, S. (1990) Monoclonal antibody Rat 401 recognizes Schwann cells in mature and developing peripheral nerve. J. Comp. Neurol., 295: 43-51.

Ince, C., Ypey, D.L., Diesselhoff-Den Dulk, M.M.C., Visser, J.A.M., De Vos, A. and Van Furth, R. (1983) Micro-CO $2^{-}$ incubator for use on a microscope. J. Immunol. Meth., 60: 269-275.

Ince, C., van Dissel, J.T. and Diesselhoff, M.M.C. (1985) A teflon culture dish for high-magnification microscopy and measurements in single cells. Pflügers Arch., 403: 240-244.

Romijn, H.J., van Huizen, F. and Wolters, P.S. (1984) Towards an improved serum-free, chemically defined medium for long-term culturing of cerebral cortex tissue. Neurosci. Bio. Behav. Rev., 8: 301-344.

Sensenbrenner, M., Lodin, Z., Treska, J., Jacob, M., Kage, M.P. and Mandel, P. (1969) The cultivation of isolated neurons from spinal ganglia of chick embryo. Z. Zellforsch., 98: 538-549.

Struwe, D.P., Wang, Z., van Graft, M., Droog, A., Ypey, D.L., Deenen, M.G.M. and Marani, E. (1991) Time-lapse recording of neonatal dorsal root ganglion (DRG) cell development in culture using a home video recorder. Eur. J. Neurosci., Suppl. 4: 279.

Trinchieri, G. (1989) Biology of natural killer cells. Adv. Immunol., 47: 187-376.

Van Dorp, R., Jalink, K., Oudega, M., Marani, E., Ypey, D.L. and Ravesloot, J.H. (1990) Morphological and functional properties of rat dorsal root ganglion cells cultured in a completely defined medium. Eur. J. Morphol., 28: 430-440. 